\title{
Multiaxial fatigue crack initiation on filled rubbers : statistical aspects
}

\author{
L. Laiarinandrasana, A. Bennani, S. Cantournet, R. Piques \\ Centre des Matériaux (UMR CNRS 7633) Ecole Nationale Supérieure des Mines de Paris BP 87, \\ F-91003 Evry Cedex - FRANCE \\ e-mail : lucien.laiarinandrasana@ensmp.fr
}

\begin{abstract}
White reinforcement fillers such as precipitated silica inclusions are traditionally used in order to increase tear resistance and reduce internal heating of technical rubber components. In this work, two precipitated silicas, which essentially differ in their specific surface area, are incorporated into a natural rubber matrix and investigated according to their mechanical behaviour and fatigue lifetime. To this end, cyclic tension/compression and torsion tests have been carried out on dogbone shaped specimens. All tests have been performed until a visible millimetric crack appears on the outer surface of the specimens. Microscopic examinations are utilised in order to find out the crack growth mechanisms in both materials. While a significant difference has been observed in terms of mechanical properties, the fatigue curves seem to merge into a unique diagram for both reinforced rubber materials. The location and the orientation of the cracks are taken into account by using the largest principal stress. Furthermore, a statistical analysis is performed based upon the amount of specific inclusions per $\mathrm{cm}^{3}$ of the rubber compound.
\end{abstract}

\section{INTRODUCTION}

This work deals with natural rubber filled with two grades of precipitated silica inclusions. The general framework of the study consists in checking all benefits of the use of this new kind of fillers in natural rubber. Hence, it is devoted to the comparison between both rubbers regarding the mechanical behaviour and fatigue lifetime. The main difference between the two grades of silica filler is their specific surface areas.

The first part of the paper deals with materials description. Then the experimental procedure is presented, beginning with the introduction of the dog-bone geometry that allows to carry out cyclic tension/compression and torsion tests. The way how to carry out the tests is then detailed. Finite Element (FE) modelling aspects are consecutively described, followed by the exploitation of the experimental database. The mechanical aspects are covered by the analysis of the local variables in the area where the cracks appear. The location and the orientation of the cracks are thus taken into account. Furthermore, a statistical analysis is performed according to the amount of noxious inclusions par $\mathrm{cm}^{3}$ of the rubber compound.

\section{EXPERIMENTS}

\subsection{Material and specimens}

The materials of interest consist of natural rubber filled with two grades of precipitated HDS. These fillers are potentially an alternative to the carbon black fillers. The material was elaborated so that both filled rubbers are supposed to be as 
similar as possible [1]. Following Bomal et al. [2], the amount of accelerator and stearic acid has been adjusted during the conventional vulcanization process. The silica fillers differ in their specific areas: respectively $113 \mathrm{~m}^{2} / \mathrm{g}$ and $189 \mathrm{~m}^{2} / \mathrm{g}$ for the corresponding materials that will be denoted as b1 and b2. The chemical composition of both materials is given in Table 1.

Table I . Formulation of b1 and b2 filled rubber materials

\begin{tabular}{|c|c|c|}
\hline Ingredient & b1 (phr) & b2(phr) \\
\hline NR & 100 & 100 \\
\hline Silica & 50 & 50 \\
\hline Silane & 4 & 4 \\
\hline ZnO & 4 & 4 \\
\hline Stearic Acid & 2 & 3.14 \\
\hline Antioxidant & 1.5 & 1.5 \\
\hline Sulphur & 1.75 & 1.75 \\
\hline Accelerator & 1.25 & 1.63 \\
\hline
\end{tabular}
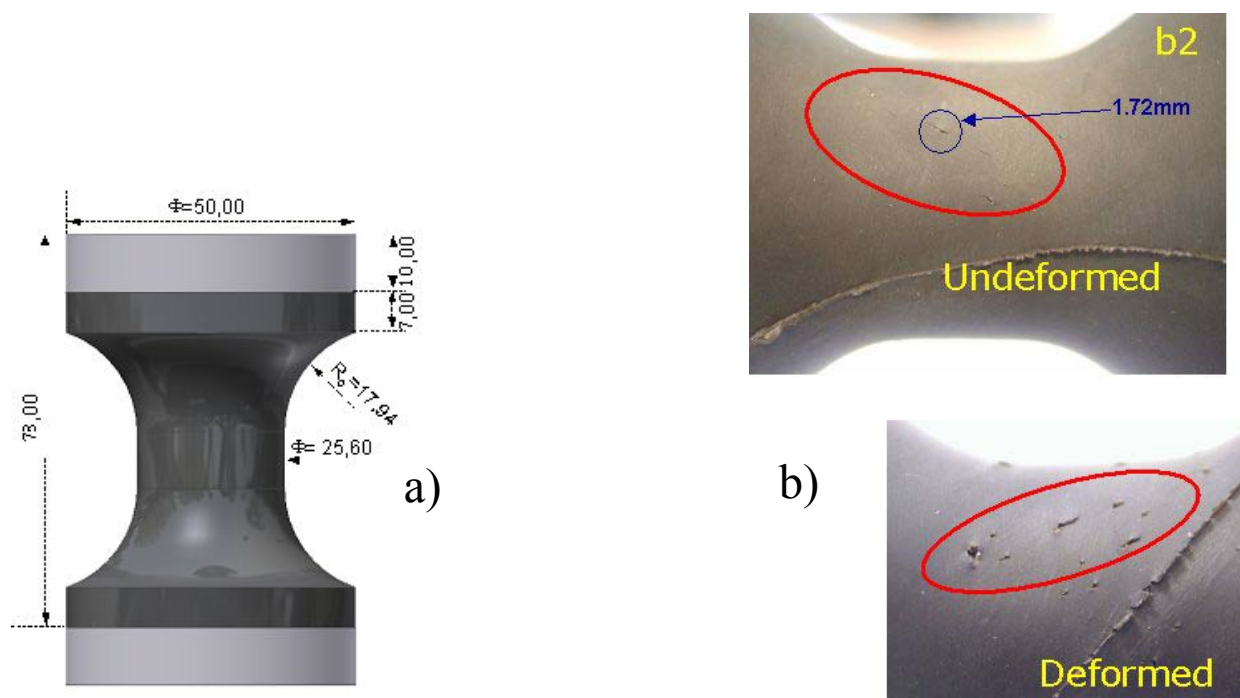

a)

b)

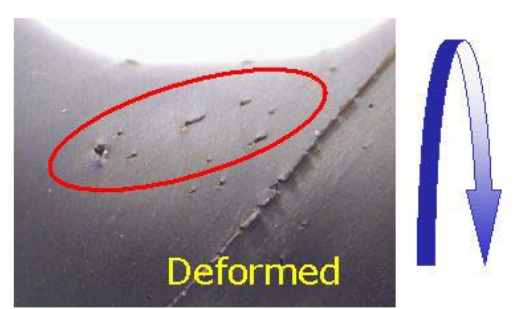

Figure 1. Dog-bone specimen a) dimensions ; b) multiple cracks under cyclic torsion

In order to compare the constitutive relationship under cyclic loads and the fatigue lifetime, a dog-bone specimen geometry has been selected (figure 1a). The advantage of this geometry is that it can be loaded by torsion (shear component) and by compression, in addition to tension. It is clear then that the stress state inside the specimen is not only multiaxial but also heterogeneous, by comparison with that of strips. For each test, the experimental data consist of load, torque, displacement and angle.

\subsection{Fatigue tests}


In this work, since we are mainly interested in comparing the fatigue lifetime of the two materials, only cyclic tests will be discussed. In [3], several cyclic tension and torsion tests were carried out. Axial displacement and rotation were imposed at the bottom end of the specimen with various mean and range values.

In order to simplify the cyclic behaviour, the stress softening due to Mullins' effects will be neglected. Additionally, to ensure that the stress relaxation due to viscoelasticity is exhausted, cycle number 1000 is considered to be representative of the stabilised materials response. Therefore, the 1000th hysteresis loops are selected to determine the cyclic mechanical behaviour of both rubber materials (figure 2).
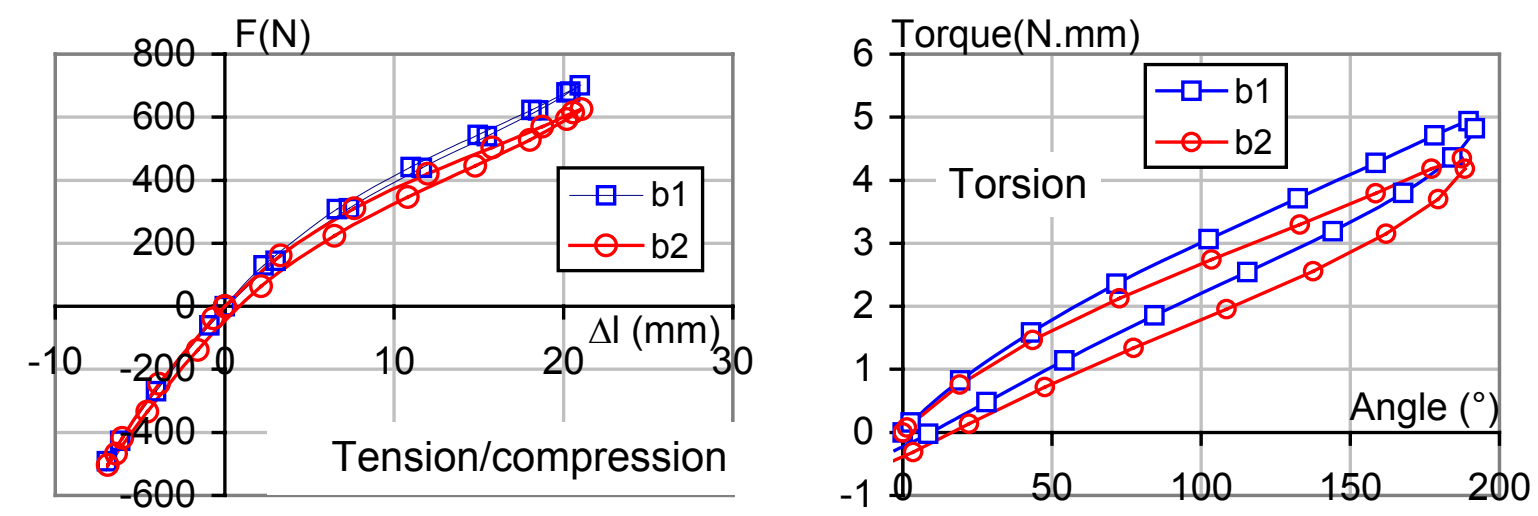

Figure 2 : Load (resp. torque) versus displacement (resp. angle) curves of both materials

In figure, the peak load and torque for b2 material are lower than that of b1. This can be attributed to the peak stress relaxation whose rate is greater for b2 compared to b1 [1]. It can then be concluded that the mechanical response of the two materials differs: b2 exhibits more dissipation (more stress relaxation and wider hysteresis loop).

\subsection{Crack initiation mechanisms}

The number of cycles to crack initiation (Ni) is supposed to be reached when a millimetric crack is optically observed on the outer surface of the specimens, so that the test is then stopped. Macroscopic and microscopic examinations reveal that several micro-cracks appear along the specimen surface for both loading conditions (see figure 1b).

The first millimetric crack is generally located next to the neck of the dog-bone specimen. At undeformed configuration, the microcracks are oriented perpendicularly to the main loading direction. Furthermore, in each examined micro-crack on the specimen surface, a coarse inclusion (about $100 \mu \mathrm{m}$ in size) is detected. Chemical analyses indicate that the inclusions responsible for crack nucleation are not silica inclusions. Unexpected elements such as $\mathrm{K}, \mathrm{Na}, \mathrm{Cl}$...were revealed. It turned out that they are already included in the chemical composition of the initial, say before elaboration, natural rubber gum. These inclusions are rather scarce but their nocivity is very high. Crack location and orientation have to be accounted for in the Finite Element (FE) modelling, whereas the presence of unexpected inclusions will be approached from a statistical viewpoint.

\subsection{Fatigue curves}


From the experimental database in [3], only tests with repeated loading are considered: displacement (resp. angle) from 0 to $L_{\max }: \Delta \mathrm{L}$ (resp. from 0 to $\theta_{\max }: \Delta \theta$ ). Therefore the fatigue curves reduce to Wöhler like diagrams. At this stage, the two modes of loading (tension, torsion) require plotting two fatigue curves: $\Delta \mathrm{L}$ (resp. $\Delta \theta$ ) versus $\mathrm{Ni}$ as illustrated in figure 3.
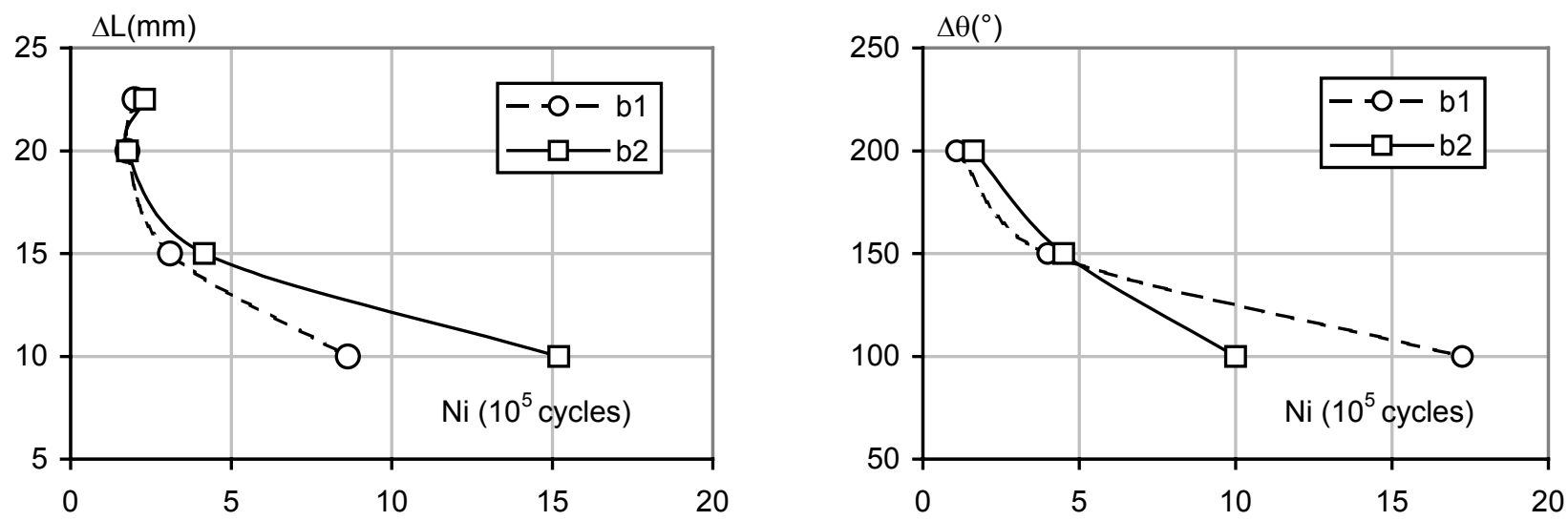

Figure 3: Fatigue curves under two kinds of imposed loading parameters $(\Delta \theta, \Delta L)$

All the abovementioned features (crack location and orientation) indicate the relevance of local approach to investigate the fatigue lifetime of both rubber materials. To this end, FE analysis is necessary to give access to the local stress and strain tensors. 3D meshes were used for cyclic torsion loading. 1536 hybrid (mixed displacement/pressure) elements have been employed in order to ensure the incompressibility constraint. For cyclic tension/compression modelling, $2 \mathrm{D}$ axisymmetric meshing is sufficient [3].

Visco-hyperelastic model was utilised to model the loop-opening phenomenon. The model is based on polynomial Rivlin deformation energy coupled with Prony series. Inverse identification of materials coefficients has been carried out, resulting in the optimised material parameters detailed in [3].
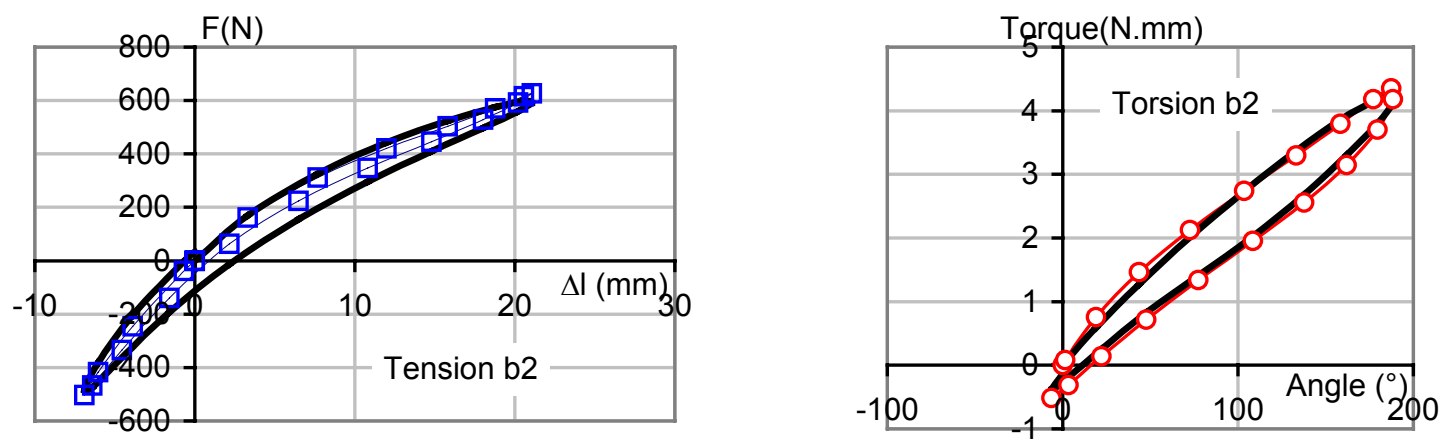

Figure 4: Comparison between experimental (symbols) and simulated (solid lines) curves for b2 material

Comparison of the simulated (solid lines) and experimental curves (symbols) is shown in figure 4 for b2 material and the following comments are also available for b1 material. Agreement is good concerning load or torque levels for the same imposed displacement or angle. Therefore, every local variable (stress or strain tensors) is expected to be correctly computed. In particular, FE results indicate that the maximum of the largest principal stress is located in areas where the first millimetric crack appears. Then, we selected one specific node belonging to this particular area. The largest principal stress is systematically computed at this node. The same approach is utilised for 2D meshes under tension/compression loading. 
The plane perpendicular to the maximum largest principal stress direction turns out to coincide with that observed on multiple crack orientation. Hence, the largest principal stress provides information concerning the location and orientation of the crack to appear. This parameter peak values are computed at the abovementioned specific node of the FE meshes.

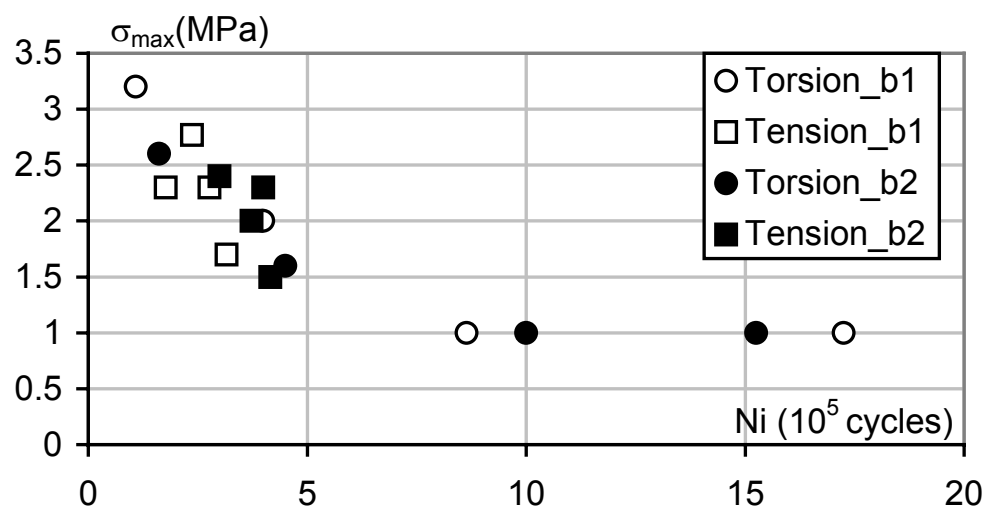

Figure 5: Unique Wöhler curve for both b1 and b2 silica filled rubber materials

Figure 5 shows for each material that tension and torsion tests merge into a unique curve. This means that the maximum largest principal stress is a relevant parameter for fatigue lifetime of both silica filled rubber materials. Additionally, both materials are represented in the same Wöhler curve. In other words, regarding the fatigue lifetime aspect, there is no difference between the two rubber materials. This is due to cavity nucleation emanating from inclusions already present in the initial natural rubber gum (the same for both materials). There might be a slight difference between both materials resistance to crack propagation, but this is negligible in comparison with the scatter inherent to Wöhler curve.

\section{Statistical aspect}

The statistical aspects are carried out by determining the mean amount of inclusions per unit volume $\alpha .$. Thus, $1 / \alpha$ corresponds to the mean volume $\mathrm{V}_{\mathrm{u}}$ containing one inclusion. By assuming that the probability to find no inclusion within a volume $\mathrm{V}$ reads:

$$
P=\exp \left(-\frac{V}{V_{u}}\right)
$$

It comes out that the volume $V_{X}$ that has the probability $X$ to contain one inclusion is :

$$
\mathrm{V}_{\mathrm{X}}=-\mathrm{V}_{\mathrm{u}} \ln (1-\mathrm{X})
$$

where In stands for Naeperian logarithm.

Void nucleation occurs as soon as the largest principal stress in the vicinity of a coarse inclusion is greater than a critical value, $\sigma_{\mathrm{c}}$. Let $\mathrm{V}_{\sigma}$ be the volume of material in the investigated geometry where the stress is greater than $\sigma_{\mathrm{c}}$. The aforementioned statistical approach can be applied by setting: $V_{X}=V_{\sigma}$. We have to find now the expression of $V_{\sigma}$.

In figure 6 , let $\mathrm{h}$ be the radius from which the largest principal stress is greater than $\sigma_{\mathrm{c}}$. For simplicity, we assume a linear distribution of the largest principal stress with respect to the radial direction. The maximum stress $\sigma_{\text {max }}$ is located at the skin of the specimen (radius $\mathrm{R}$ ). We are concerned with the volume $\mathrm{V}_{\sigma}$ inside which the largest principal stress value lays between $\sigma_{\mathrm{c}}$ and $\sigma_{\max }$. 


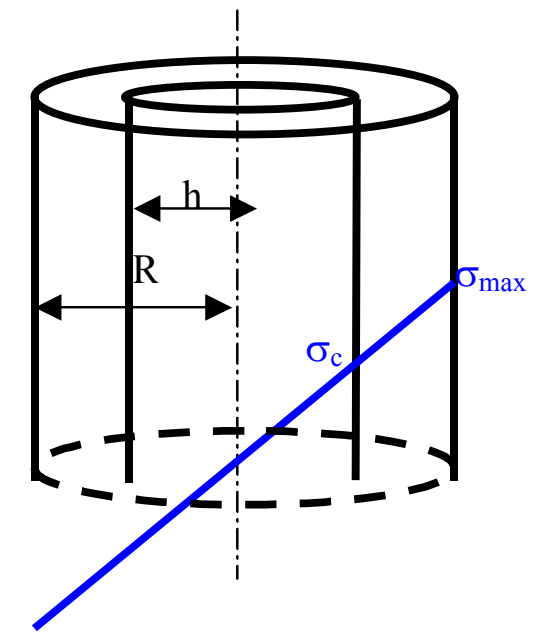

Figure 6. Sketch illustrating the volume $\mathrm{V}_{\sigma}$

Eq. 3 can be also written as follows :

$$
\frac{\mathrm{V}_{\sigma}}{\mathrm{V}_{\text {total }}}=1-\frac{\mathrm{V}_{\mathrm{h}}}{\mathrm{V}_{\text {total }}}
$$

With ,

$$
\frac{V_{h}}{V_{\text {total }}}=\frac{\pi h^{2} L}{\pi R^{2} L}=\left(\frac{h}{R}\right)^{2}
$$

The assumption of linear distribution of the largest principal stress with respect to the radial direction yields:

$$
\frac{\sigma_{\mathrm{c}}}{\sigma_{\max }}=\frac{\mathrm{h}}{\mathrm{R}}
$$

Eq. 5 together with eq. 6 lead to:

$$
\frac{\mathrm{V}_{\mathrm{h}}}{\mathrm{V}_{\text {total }}}=\left(\frac{\sigma_{\mathrm{c}}}{\sigma_{\max }}\right)^{2}
$$

Now back to $V_{X}=V_{\sigma}$ reads :

$$
-V_{u} \ln (1-X)=V_{\text {total }}\left(1-\left(\frac{\sigma_{c}}{\sigma_{\max }}\right)^{2}\right)
$$

Eq 8 gives the expression of $\sigma_{\max }$ :

$$
\sigma_{\max }=\frac{\sigma_{\mathrm{c}}}{\sqrt{1+\frac{\mathrm{V}_{\mathrm{u}}}{\mathrm{V}_{\text {total }}} \ln (1-\mathrm{X})}}
$$

Since $V_{u}=1 / \alpha$, eq. 9 can be also written like this : 


$$
\sigma_{\max }=\frac{\sigma_{\mathrm{c}}}{\sqrt{1+\frac{\ln (1-\mathrm{X})}{\alpha \bigvee_{\text {total }}}}}
$$

$\mathrm{V}_{\text {total }}$ depends on the specimen size. For the dogbone specimen in figure 1,

$$
\pi R^{2} L \approx \pi(1.28)^{2}(1) \approx 5 \mathrm{~cm}^{3}
$$

$\alpha$ depends on the material. Unfortunately, the amount of the inclusions per $\mathrm{cm}^{3}$ has not been estimated at present time. In order to overcome this difficulty, the following development will be first parameterized by $\alpha$. The relevance of the adjusted value of $\alpha$ will then be discussed.

Let us now consider a given number of cycles to crack initiation on the Wöhler curve (fig. 5). The probability of crack initiation is null if the largest principal stress at the surface is less than $\sigma_{\mathrm{c}}$. Hence, the lower bound of the Wöhler curve represents the $\sigma_{c}$ value corresponding to the given number of cycles to crack initiation.

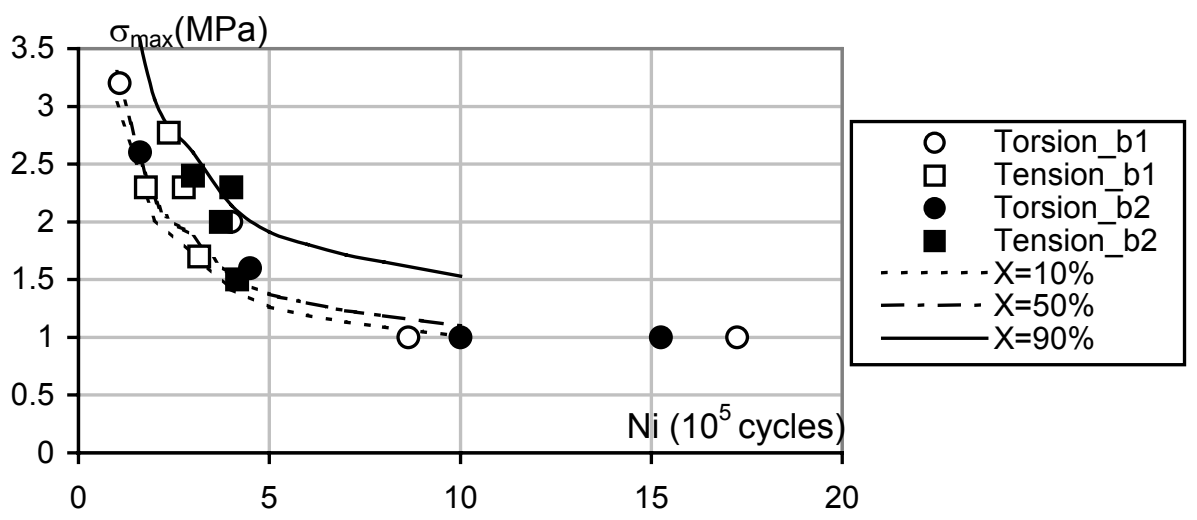

Figure 7 : Wöhler curve with the upper and lower bound modelled by $a=0.8 / \mathrm{cm} 3$.

The exploitation of eq. 10 consists in identifying for each number of cycles to crack initiation in figure 5 the amount of $\alpha$ allowing to model the scatter band. After several trials, the computation resulted in $\alpha=0.8$ inclusion per $\mathrm{cm}^{3}$. Figure 7 illustrates the lines with respectively probabaility of $10 \%, 50 \%$ and $90 \%$.

It is to be mentioned that there is very slight difference between $10 \%$ and $50 \%$, meaning that $50 \%$ of the probability is not related to the "best fit curve" corresponding to the average one between $10 \%$ and $90 \%$. In addition, it has been observed that these bounds are too sensitive to the $\alpha$ value. For instance, when $\alpha=10$ inclusions per $\mathrm{cm}^{3}, \sigma_{\max }(10 \%)=1.001 \sigma_{\mathrm{c}}$ and $\sigma_{\max }(90 \%)=1.02 \sigma_{\mathrm{c}}$, whereas for $\alpha=0.5$ inclusion per $\mathrm{cm}^{3}, \sigma_{\max }(10 \%)=1.02 \sigma_{\mathrm{c}}$ and $\sigma_{\max }(90 \%)=3.55 \sigma_{\mathrm{c}}$. In our opinion, it is due to the fact that other probabilities have to be accounted for in the present model: the probability of neighbouring (inclusion clustering or the surface neighbouring), the inclusion size distribution etc...So the probability aspect presented here only gives a partial explanation to the scatterband.

\section{CONCLUSION}

The materials of interest labelled b1 and b2 consist of natural rubber filled with two grades of precipitated silica which differ in their total surface specific area. Dog-bone specimens have been tested under two kinds of loading conditions until visible millimetric crack is detected: cyclic tension/compression with prescribed displacement and cyclic torsion with prescribed rotation (angle).

Microscopic examinations are utilised in order to find out the location and the orientation of the crack and to depict the damage mechanisms. Void nucleation occurrs at the interface between coarse inclusions (other than silica) and rubber matrix. 
The 1000th cycle has been considered to represent the stabilised response of the material. A visco-hyperelastic model based on generalised Rivlin strain energy and Prony series has been used. The local maximum largest principal stress coincides with the location where the crack has been experimentally detected. It is then systematically computed in order to assess the fatigue lifetime.

The fatigue lifetime of both silica filled rubber materials has been characterized first with $\Delta \mathrm{L}($ resp. $\Delta \theta)$ versus $\mathrm{Ni}$ diagrams. By using the peak values of the largest principal stress, the latter diagrams merge into a unique Wöhler curve. This shows the relevance of the largest principal stress for fatigue crack initiation prediction. Furthermore, since fatigue damage emanates from coarse inclusions already included in the rubber matrix, the same Wöhler curve is available for both materials.

An attempt is made to utilise a simple statistical approach based on the amount of deleterious inclusions to predict the scatter band in the unique Wöhler curve.

\section{REFERENCES}

[1] Bennani A., Laiarinandrasana L, Piques R. and Cantournet S., "Influence of filler properties on the mechanical response of silica filled natural rubber", Proceedings of the third european conference on constitutive models for rubber (ECCMR) London pp. 177-184; 2003.

[2] Bomal Y., Cochet P. and Dejean B., "Influence of specific surface area and quantity of precipitated silica on the properties of a precipitated silica filled natural rubber", in Meeting of rubber division, American Chemistry Society, Denver paper $39 ; 1993$.

[3] Laiarinandrasana L., Bennani A., Cantournet S. and Bomal Y., "Mechanical behaviour and fatigue lifetime of natural rubber reinforced by two grades of precipitated silica", Proceedings of the fourth european conference on constitutive models for rubber (ECCMR) Stockhölm pp. 109-114; 2005. 